\title{
The Effects of Soothing Techniques and Rough-and-Tumble Play on the Early Development of Temperament: A Longitudinal Study of Infants
}

\author{
Atsuko Nakagawa and Masune Sukigara \\ Graduate School of Humanities and Social Sciences, Nagoya City University, 1 Yamanohata, Mizuho-cho, Mizuho-ku, \\ Nagoya 467-8501, Japan \\ Correspondence should be addressed to Atsuko Nakagawa; nakagawa@hum.nagoya-cu.ac.jp
}

Received 26 February 2014; Revised 27 June 2014; Accepted 4 August 2014; Published 20 August 2014

Academic Editor: Glenda Andrews

Copyright (c) 2014 A. Nakagawa and M. Sukigara. This is an open access article distributed under the Creative Commons Attribution License, which permits unrestricted use, distribution, and reproduction in any medium, provided the original work is properly cited.

\begin{abstract}
A total of 189 infants (93 girls, 96 boys) were investigated longitudinally at ages 4, 8, 12, 18, and 24 months to examine the effects of soothing techniques (i.e., distracting infants by presenting novel objects) and rough-and-tumble play on the early development of temperament, particularly the emergence of Effortful Control. We used questionnaires to examine the frequency of use of soothing techniques and rough-and-tumble play. The Infant Behavior Questionnaire Revised (IBQ-R) and the Early Childhood Behavior Questionnaire (ECBQ) were used to assess temperament. A strong relationship was found between parental ratings of their infant's Orienting/Regulation and later Effortful Control. Caregivers' use of distracting as a soothing technique during infancy was associated with higher Negative Affect in toddlers at 24 months. More surgent infants were involved in more rough-and-tumble play, with rough-and-tumble play frequencies positively correlated with surgency scores at 24 months.
\end{abstract}

\section{Introduction}

Considerable progress has been made recently in understanding the attention system of the human brain and its consequences for human development. Ruff and Rothbart [1] argued for attention to be seen as part of the mechanisms of self-regulation or the ability to modulate behavior according to the cognitive, emotional, and social demands of specific situations. Posner and colleagues defined three attentional networks-alerting, orienting, and executive-that underlie achieving and maintaining a state of high sensitivity to incoming stimuli, aligning attention with sources of sensory signals, and monitoring and resolving conflicts among thoughts and ideas $[2,3]$.

The relationship between the development of attentional networks and the regulation of emotion has been emphasized very early in life from the perspective of temperament [4]. Temperament is defined as constitutionally based individual differences in reactivity and self-regulation in the domains of emotion, activity, and attention [5]. Recent evidence suggests that during infancy, control may involve primarily the brain's orienting attention network, including the parietal lobe and frontal eye fields [6]. The efficiency of this orienting network develops dramatically in the first year of life (e.g., [7-9]). By 3 to 4 years of age, control may reflect more strongly a frontal executive attention network that involves the anterior cingulate and basal ganglia. During infancy, the external environment, including caregivers, seems to exercise much of the control, while older children and adults are guided by internal goals. There is also empirical evidence for this shift because early evidence of self-regulation during infancy (e.g., looking away and self-soothing) correlates with the orienting network as measured by the attention network test at age 7 $[10,11]$.

The development of the orienting attentional network can be documented through tasks that measure the infant's ability to disengage from a location [12]. Johnson et al. [9] investigated individual differences in disengagement using a gap-overlap task and reported that those 4-month-old infants who disengaged more easily were less susceptible to distress and easier to soothe, as reported by their parents. In a gapoverlap task, a central fixation stimulus is presented prior to 
the appearance of a peripheral stimulus. The central fixation may remain on the monitor after the peripheral target is presented (the overlap condition), or it may disappear for a brief interval prior to the presentation of the peripheral stimulus (the gap condition). Between 2 and 4 months, the ability to disengage in the overlap condition develops very rapidly $[13,14]$. The early development of the disengagement operation is of particular interest as it may play an important role in the early regulation of emotion (e.g., [15-18]).

Individual differences in the efficiency of the early orienting network have also been examined by applying a parent-completed measure of child temperament, the Infant Behavior Questionnaire Revised (IBQ-R; [19]). Factor analyses of scale scores in IBQ-R yielded three broad factors of temperament in infants aged 3 to 12 months. As distinct from the two broad factors of Surgency and Negative Affect, a third factor called Orienting/Regulation was extracted. Furthermore, from toddlerhood and beyond, this same third factor likely represents the Effortful Control dimension in a series of behavioral questionnaires (such as the Early Childhood Behavior Questionnaire (ECBQ; [20])) that measure the operation of the executive attention system [21]. Effortful Control refers to the ability to inhibit a dominant response in order to perform a subdominant response, detect errors, and engage in planning. Longitudinal studies using IBQ-R for infants and ECBQ for toddlers have revealed that Orienting/Regulation as assessed in infancy is related to levels of Effortful Control in toddlers [22-24].

Rothbart et al. [4] noted that how infants are soothed might influence the development of connections between the orienting and executive networks. One such soothing technique involves distracting the infant by drawing his or her attention to novel objects. That is, novel objects presented by the caregiver might help the infant disengage attention from the current distress. Harman et al. [25] found that distress induced by experimental overstimulation in 3-and 6-month old infants could be remediated by the presentation of novel objects. Posner and Rothbart [12] suggest that during occasions where infants are momentarily soothed by distractions, caregivers train the infants to develop control over their emotions.

Regarding the effects of interchange with caregivers on the development of attention, Bornstein [26] reported that infants who processed visual information more efficiently (i.e., those who habituated more quickly) and had mothers who more frequently encouraged them to attend to properties, objects, and events in the environment were superior in verbal development during their second year and obtained higher scores on an intelligence test at 4 years of age. Akin to the results of Bornstein [26], mothers who more frequently distracted their infant (i.e., encouraged him or her to disengage from current distress) by orienting his or her attention to novel stimuli during soothing might influence the child's future attentional control system. However, it is also reported that as soon as the infant's orientation to the novel object or event is lost, the infant's distress returns to the level shown prior to the presentation of the distractor [25]. Because there are anatomical reasons why the regulation of emotion may pose a difficult problem for a child [27], soothing techniques aiming to distract or early approaches to emotion regulation might be different from the maternal encouragement of attention during times when the child is likely to be in a state of quiet or active alert, as assessed by Ruddy et al. [28] and Tamis-LeMonda and Bornstein [29].

According to Panksepp [27], rough-and-tumble play could also influence the development of temperament as a potential facilitator of Positive Affect. Rough-and-tumble play, which taps into the physical social engagement system, is a fundamental form of play that naturally leads to chasing, romping, and wrestling and is accompanied fairly consistently by Positive Affects, as signaled by joyous shrieking and laughter. Furthermore, as with the early affect control system, Panksepp [30] suggests that without adequate play (i.e., rough-and-tumble play), self-control and other executive functions may not properly mature. As our previous study investigated the relationship between rough-and-tumble play and temperament in toddlers [31], in the present study, we study this relationship from infancy longitudinally.

Our goal is to examine the influence of how infants are soothed on the early development of the attentional regulatory capacity in light of temperament. We longitudinally administered temperament questionnaires (IBQ-R and ECBQ) together with questionnaires assessing the soothing methods used by parents and the type of play accompanied by Positive Affect, including smiling and laughing. We hypothesized that children whose mothers more frequently present distractors during soothing or who are involved in more rough-and-tumble play will have higher Effortful Control scores on the ECBQ at 24 months.

\section{Method}

2.1. Participants. We recruited a sample of caregivers with infants between 3 and 4 months of age from the community in Nagoya, Japan's third largest industrial metropolis in the center of the country. According to the 2010 national census under the control of Ministry of Internal Affairs and Communications, its total population is 2,263,894, spread over $326.43 \mathrm{~km}^{2}$. The 2010 national census also shows that $75.5 \%$ of the working population is employed in the tertiary sector of the economy (also known as the "service sector"), with the remainder working in the manufacturing sector $(24.3 \%)$, or the agriculture, forestry, or fishery sectors. According to a 2012 dataset under the control of Ministry of Health, Labor, and Welfare, $2.1 \%$ of children born in Japan were born out of wedlock. In the present study, $100 \%$ of the caregivers were Japanese.

We asked caregivers to take part in this investigation of their infants' temperaments while they were visiting public health centers for their infants' routine 3-month medical examination, which is offered free of charge by municipalities in Japan and where a height and weight measurement, an internal medicine medical examination, and a child-care consultation are conducted. Caregivers with twins were not included. We provided questionnaires to 247 caregivers who agreed to participate at 4 months, with further questionnaires 
subsequently mailed to the participants' homes at 8 months (242 caregivers), 12 months (235 caregivers), 18 months (226 caregivers), and 24 months (217 caregivers). The caregivers were primarily female (98\%), except for two males and one unknown, and the infants consisted of 96 boys and 93 girls. Only families with healthy full-term infants and for whom we had complete questionnaires at all five assessment points were eligible for the current analysis, resulting in a total of 189 caregivers in the sample, with an average age 30.17 $(\mathrm{SD}=8.52)$. At the start of the study, about $56.6 \%$ of the children in the sample were first-born, $34.9 \%$ second-born, $7.9 \%$ third-born, and $0.5 \%$ fourth-born, with $8.4 \%$ of children experiencing the arrival of a younger sibling in their first two years. Participants responding five times over the course of the study were given a book token worth 5,000 yen (approximately $\$ 50$ ).

\subsection{Temperament Questionnaires}

Infant Behavior Questionnaire Revised (Japanese IBQ-R [32]). This 191-item parent report instrument, which is designed for children between 3 and 12 months of age, contains 14 subscales that yield three broad factors: (a) Surgency, which includes activity level, approach, high-intensity pleasure, perceptual sensitivity, smiling and laughter, and vocal reactivity as subscales; (b) Negative Affect, which includes distress to limitations, fear, sadness, and the negatively loaded falling reactivity as subscales; and (c) Orienting Control, which includes duration of orienting, soothability, cuddling/affiliation, and low-intensity pleasure.

Early Childhood Behavior Questionnaire (Japanese ECBQ [33]). This 201-item measure contains 18 subscales, which also yield three overarching factors: (a) Surgency, which includes impulsivity, activity level, high-intensity pleasure, sociability, and positive anticipation as subscales; (b) Negative Affect, which includes discomfort, fear, sadness, frustration, motor activation, perceptual sensitivity, shyness, and the negatively loaded soothability as subscales; and (c) Effortful Control, which includes inhibitory control, attention shifting, lowintensity pleasure, cuddling, and attention focusing as subscales. The ECBQ is designed for children between 18 and 36 months of age.

Caregivers were asked to report the frequency of specific child behaviors on a 7-point Likert-style scale ranging from 1 ("never") to 7 ("always"), in the past week for the IBQ-R and in the past two weeks for a portion of the IBQ-R (two weeks for less frequent events during the first year) and the ECBQ. Adequate internal consistencies (Cronbach's alpha) for both questionnaires were reported in Nakagawa and Sukigara [32] and Sukigara et al. [33]. Based on our previous data, scalebased and item-based alphas for the IBQ-R factors were .838 and .943 for Surgency, .627 and .899 for Negative Affect, and .510 and .734 for Orienting/Regulation. Corresponding alphas for the ECBQ were .620 and .875 for Surgency, .751 and .913 for Negative Affect, and .654 and .893 for Effortful Control.
Soothing Method Questionnaire. The caregivers were given 20 situations in which children would show Negative Emotionality and asked to select one of five choices describing the behavior they might opt for in each situation: (a) cuddling/giving love; (b) distracting/attracting the child's attention to a novel object or event; (c) giving drinks or snacks; (d) waiting for the child to quiet down without assistance; and (e) not soothing the child because he or she does not cry or grumble. Of the 20 situations, two are related to physical pain (e.g., "if your child starts crying when given an injection"), four to fear (e.g., "if your child cries when he or she is startled by loud noises (such as a fire truck siren) "), eight to frustration (e.g., "if your child gets irritated when being told that it is time for bed or a nap"), five to sadness (e.g., "if your child becomes sad when you say NO"), and one to discomfort (e.g., "if your child seems to be bothered by sounds in noisy environments"). These situations were related to items in Nakagawa and Sukigara [31]. Frequencies for each of the five choices were calculated per respondent.

Type of Play Questionnaire. The caregivers were given one A4sized sheet illustrating 34 frequent play activities, including five rough-and-tumble plays (i.e., jumping with support on both sides, chasing, tickling, being lifted up, and being rolled over). They were asked to choose up to five forms of play that make their child laugh out loud, as children should be smiling or laughing while engaged in rough-and-tumble play. For each choice, they were asked to assess the frequency of such play (a: frequently, b: sometimes, c: occasionally).

\section{Results}

3.1. Temperament, Play, and Soothing Method Questionnaires. Means and standard deviations for IBQ-R or ECBQ scores for the temperament-related factors at each age are shown in Table 1. There were significant age effects (within subject) on all three temperament scores (Surgency: $F(4,752)=$ 256.6, Negative Affect: $F(4,752)=91.7$, Orienting/Effortful Control: $F(4,752)=105.1, p s<.001)$. Older infants obtained higher scores on Surgency. Negative Affects also increased in the first year of life. However, younger infants' scores were higher for Orienting/Effortful Control. These tendencies were consistent with previous studies $[19,20]$.

Based on the questionnaire for type of play, we divided participants into rough and nonrough groups such that if the first two choices included any rough-and-tumble activities (i.e., jumping with support on both sides, chasing, tickling, being lifted up, or being rolled over) evaluated to occur frequently, these cases were categorized into the rough group, with the remainder of cases categorized into the nonrough group. Table 1 also shows the percentage of participants in each group. We found a significant effect of age $\left(\chi^{2}=96.7\right.$, $d f=4, p<.001)$. Throughout the first year of life, more than half of the participants were categorized into the rough group. Then as toddlers become able to enjoy a rich variety of play, the percentage in the rough group decreased. The top six play activities in terms of total frequency for each play at each age are presented in Table 2. We included a maximum of three choices per caregiver as most participants gave only 
TABLE 1: Means (SD) for temperament scores and percentages (number of participants) categorized by play type.

\begin{tabular}{lccccc}
\hline & & IBQ-R & & ECBQ \\
& 4 months & 8 months & 12 months & 18 months & 24 months \\
\hline Positive affectivity/Surgency & $3.62(.69)$ & $4.30(.57)$ & $4.53(.54)$ & $4.81(.65)$ & $4.95(.62)$ \\
Negative Emotionality & $3.11(.66)$ & $3.59(.66)$ & $3.66(.60)$ & $3.00(.48)$ & $4.15(.54)$ \\
Regulatory capacity/orienting & $4.75(.60)$ & $4.55(.55)$ & $4.52(.48)$ & $4.05(.54)$ & \\
\hline & & & & \\
& 4 months & 8 months & 12 months & 18 months & 24 months \\
\hline Rough \% $(N)$ & $60.3(114)$ & $83.1(157)$ & $73.5(139)$ & $50.3(95)$ & $39.7(75)$ \\
Nonrough \% $(N)$ & $39.7(75)$ & $16.9(32)$ & $26.5(50)$ & $49.7(94)$ & $60.3(114)$ \\
\hline
\end{tabular}

three responses on the questionnaire. Tickling is recognized as a typical playful situation, with laughter occurring across all ages.

Choice ratios for the 20 questionnaire items assessing five different soothing methods were calculated per respondent (\%). Table 3 presents each mean choice for all 189 participants at each age. To deal with the uneven distribution, an arcsin transformation was applied to the data. Following a onefactor ANOVA, the main effect of age (within subject) was significant for all choices, from (a) to $(\mathrm{e}): F(4,752)=2.40$, $\left.p<.05 ; F_{s}(4,752)=48.33,3.54,47.53,52.95, p s<.01\right)$. Choice (b) (distracting/attracting the child's attention to a novel object or event) increased during the first year of life. Activity (c) (giving drinks or snacks) is the least applied at 4 months. Choice (d) (leaving him/her as he/she is) gradually increased, while choice (e) (not soothing the child because he or she does not cry or grumble) decreased with months of age.

3.2. Relationships between Soothing Method, Type of Play, and Temperament. A path analysis was conducted using Amos software (ver. 16) to clarify the relationships between soothing method, type of play, and temperament (Surgency, Negative Affect, and Orienting/Effortful Control) across the 4- to 24month longitudinal data. With regard to soothing method, the index of cuddling/giving love or distracting refers to the sum of ratios (following an arcsin transformation) of choices (a) or (b) at 4 months, 8 months, and 12 months. Frequencies for inclusion in the rough group were calculated from 4 months through to 12 months. That is, if the child was classified as belonging to the rough group three times (at 4,8 , and 12 months), he or she was evaluated as 3 (range: $0-3)$. When we checked for multivariate normality using Mardia's test $[34,35]$, both skewness and kurtosis values did not indicate multivariate normality. Using robust estimates of Mahalanobis distances, multivariate outliers $(n=9)$ were removed, following which Mardia's coefficient indicated multivariate normality, as follows: skewness $\left(6.03, \chi^{2}=\right.$ $180.90, p=.18)$ and kurtosis $(97.54, z=-0.69, p=.48)$. The chi-square probability plot for our data $(N=180)$ is shown in Figure 1.

The hypothesized model (Figure 2) was designed to investigate the paths from temperament scores at 4 months to those at 24 months, considering effects of soothing method and rough-and-tumble play. The Bentler comparative fit

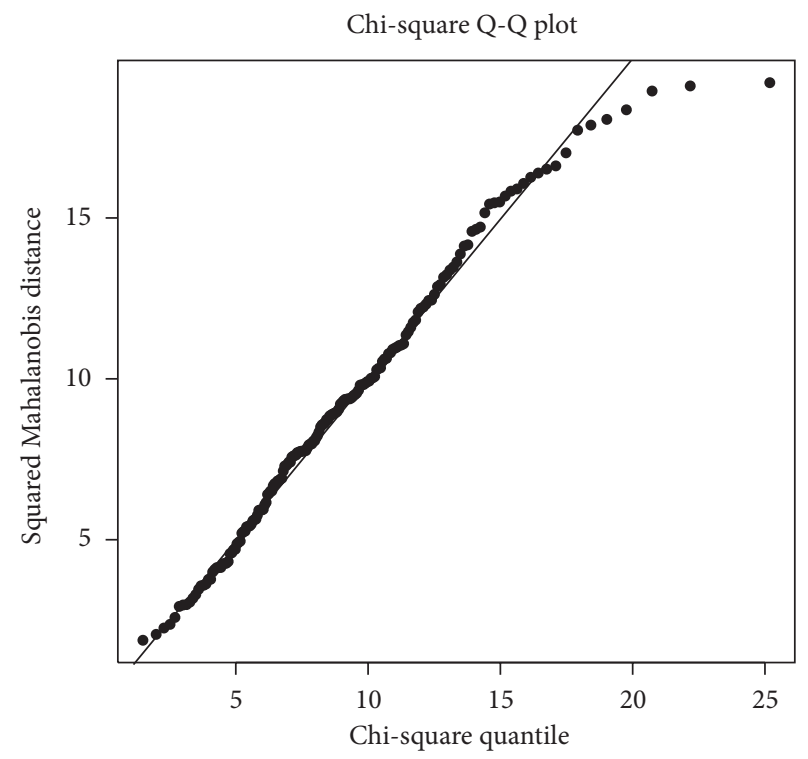

FIGURE 1: Chi-squared quantile-quantile plot for the data assessing multivariate normality $(N=180)$.

index (CFI) was 0.957, and the root mean square error of approximation (RMSEA) value was .046. As expected, Surgency, Negative Affect, and Orienting/Effortful Control were highly stable throughout infancy and toddlerhood, even though temperament questionnaire scores changed between 12 and 18 months.

We found that the extent of giving love or cuddling could result from the degree of Negative Affect and Surgency shown by the infants, with a $b$ weight of .19 and .23 , respectively. The frequency of giving love or cuddling appears to influence the development of Negative Affect, with a $b$ weight of .14. Similarly, caregivers' use of distraction in infancy contributed to higher Negative Affect at 24 months, with a $b$ weight of .16 , as it was elicited by Surgency, with a $b$ weight of .20. In addition, consistent with our previous study [31], we found a relationship between rough-and-tumble play and temperament such that rough-and-tumble play in infancy had a positive effect on Surgency at 24 months, with a $b$ weight of .15. The degree of Surgency at 4 months may also have contributed to the frequency of rough-and-tumble play, with a $b$ weight of .21 . 
TABLE 2: Total frequencies for each play activity (top six activities for each age, with a maximum of three choices per caregiver).

\begin{tabular}{|c|c|c|c|c|c|}
\hline \multirow{2}{*}{ Playing } & \multicolumn{5}{|c|}{ Total frequency at each age } \\
\hline & 4 months & 8 months & 12 months & 18 months & 24 months \\
\hline Tickling & 132 & 149 & 113 & 107 & 84 \\
\hline Peekaboo & 85 & 75 & 68 & 67 & \\
\hline Being lifted up & 78 & 114 & 75 & 72 & \\
\hline Jumping with support on both sides & 77 & 117 & 37 & 32 & \\
\hline Hand games & 16 & & & & \\
\hline Listening to music & 15 & & & & \\
\hline Chasing & & 18 & 66 & 47 & \\
\hline Watching TV/DVD & & 13 & & & 30 \\
\hline Dancing to music & & & 22 & 34 & 53 \\
\hline Sliding by oneself/with a caregiver & & & & & 40 \\
\hline Playing horsey & & & & & 31 \\
\hline Jumping & & & & & 27 \\
\hline
\end{tabular}

Bold indicates rough-and-tumble play activities.

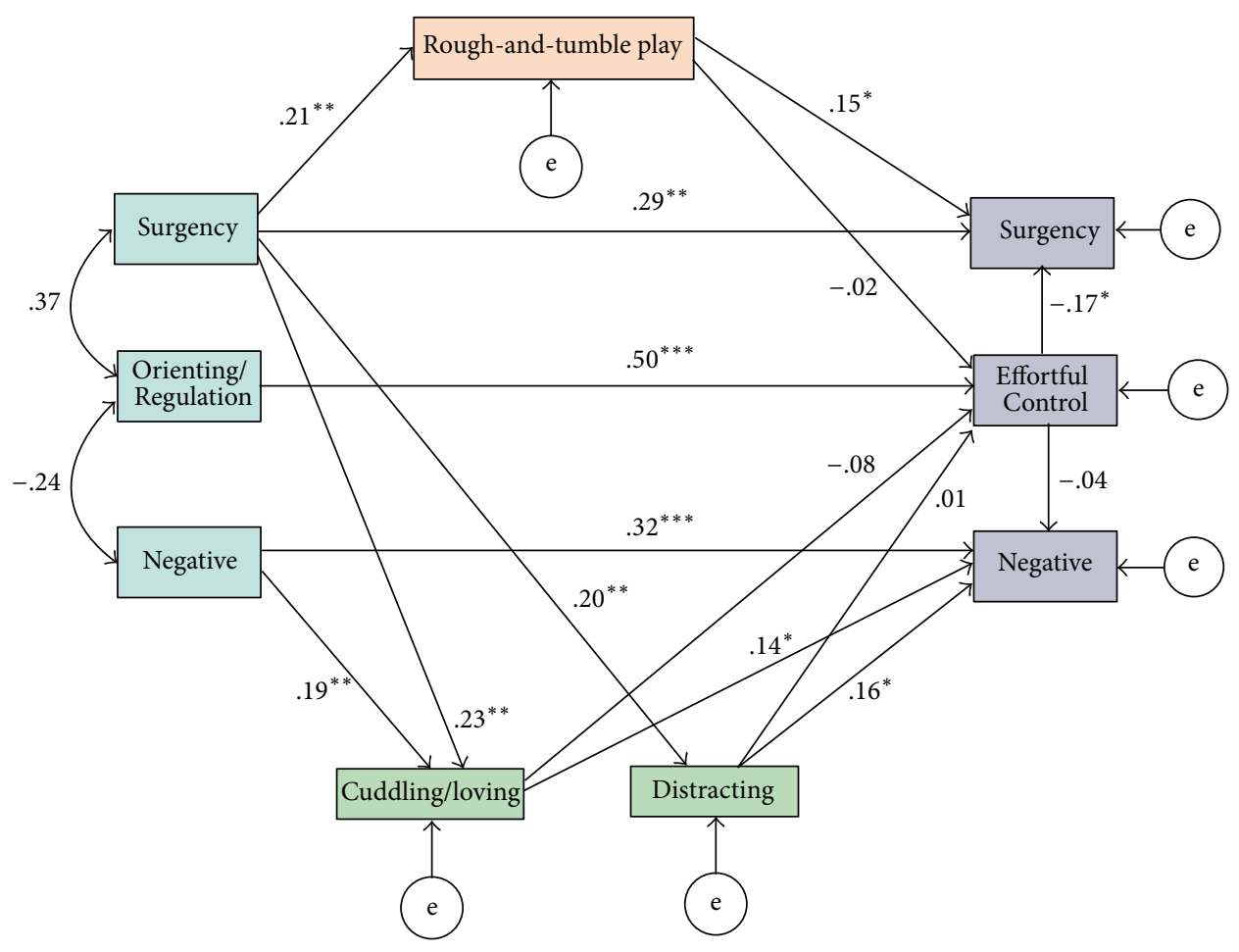

24 months

4 months

FIGURE 2: Path diagram of causal relationships between temperament, soothing method, and rough-and-tumble play between infancy and toddlerhood. Coefficients are standardized beta values. Note: ${ }^{* * *} p<.001 ;{ }^{* *} p<.01 ;{ }^{*} p<.05$.

To test the significance of two indirect effects, the RMediation software [36] was used to examine $95 \%$ confidence intervals (CIs) for each of the two-path indirect effects. For the indirect effect of 4-month Surgency on 24-month Surgency via rough-and-tumble play, the $95 \%$ CI was [.001, .076]. For the indirect effect of 4-month Negative Affect on 24-month Negative Affect via cuddling/loving, the 95\% CI was $[0, .051]$. The results suggest that only 4 -month Surgency had significant and indirect effects on 24-month Surgency via rough-and-tumble play, while 4-month Negative Affect had marginally significant and indirect effects on 24-month Negative affects via cuddling/loving.

3.3. Relationship between the Situation and Soothing Methods. To clarify the situation linked to the caregivers' use of distracting soothing for further studies to investigate the effect of 
TABLE 3: Means (SD) for choice ratios of five soothing activities at each age.

\begin{tabular}{|c|c|c|c|c|c|}
\hline Variable & 4 months & 8 months & 12 months & 18 months & 24 months \\
\hline (a) Cuddling/giving love & $.39(.22)$ & $.43(.19)$ & $.41(.16)$ & $.42(.18)$ & $.40(.16)$ \\
\hline (b) Distracting/attracting his/her attention to a novel object or event & $.14(.15)$ & $.25(.14)$ & $.29(.15)$ & $.28(.14)$ & $.29(.15)$ \\
\hline (c) Giving drinks or snacks & $.007(.023)$ & $.013(.027)$ & $.015(.027)$ & $.014(.027)$ & $.012(.026)$ \\
\hline (d) Leaving him/her as he/she is & $.07(.11)$ & $.09(.12)$ & $.11(.11)$ & $.16(.12)$ & $.18(.12)$ \\
\hline (e) Not soothing the child as he/she does not cry/grumble & $.38(.39)$ & $.23(.24)$ & $.18(.19)$ & $.13(.13)$ & $.13(.13)$ \\
\hline
\end{tabular}

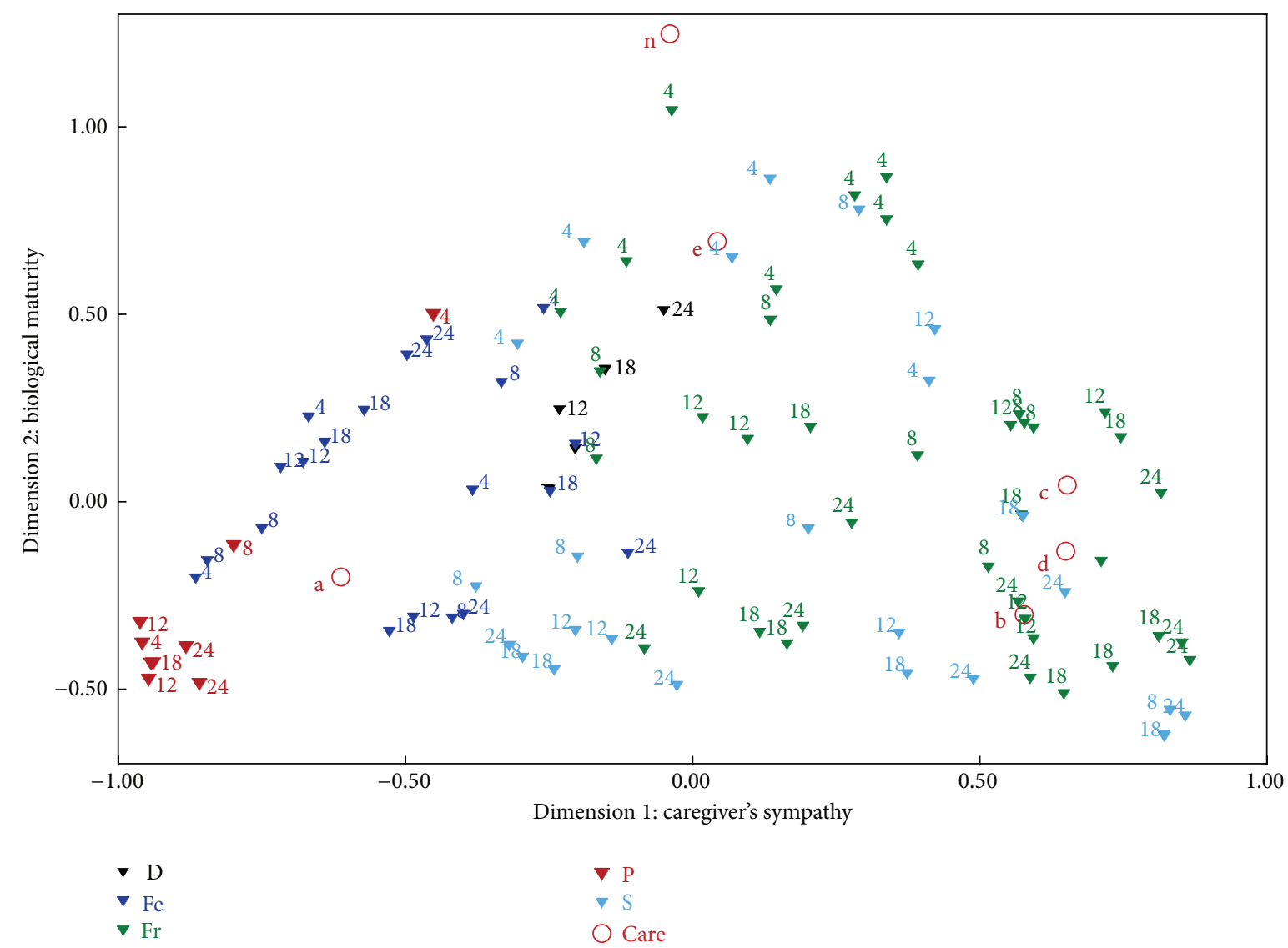

FIGURE 3: Correspondence analysis solution for soothing method and situations (Dimensions 1 \& 2). Note: D: discomfort; Fe: fear; Fr: frustration; P: physical pain; S: sadness; a: cuddling/giving love; b: distracting/attracting his/her attention to a novel object or event; c: giving drinks or snacks; d: waiting for the child to quiet down without assistance; e: not soothing the child because he/she does not cry or grumble; n: no answer. Each number indicates months of age.

distraction method, we performed correspondence analyses on data from the soothing method questionnaire at all five assessment points. The eigenvalues for the first three principal axes were $.535, .411$, and .350 , with cumulative contribution rates of .432, .690, and .876, respectively. Figures 3 and 4 show the scatter plots of twenty situations for children's Negative Affect for each age and soothing categories on a two-dimensional map. In Figure 3, these are presented with Dimension 1 on the horizontal axis and Dimension 2 on the vertical axis. In Figure 4, Dimension 1 is on the horizontal axis and Dimension 3 is on the vertical axis. Each of five red letters $(\mathrm{a}-\mathrm{e})$ with a red outline circle indicates the five soothing methods, while " $n$ " stands for "no answer." The other five colored markers indicate each situation corresponding to the questionnaire items: D for discomfort, Fe for fear, Fr for frustration, $\mathrm{P}$ for physical pain and $\mathrm{S}$ for sadness. For each age, these 20 markers were plotted with a small number of months of age.

In Figures 3 and 4, the horizontal direction of the plot (the first dimension) divides situations according to caregivers' sympathetic reactions to their infants' negative expressions. The situations on the left are associated with giving love or cuddling, while situations on the right are associated with less sympathetic responses such as distracting, ignoring, or turning away from the child. Situations linked to physical pain or fear are also represented at the left pole on the first dimension. Turning to the vertical direction (the second dimension) in Figure 3, the categories of no response or not 


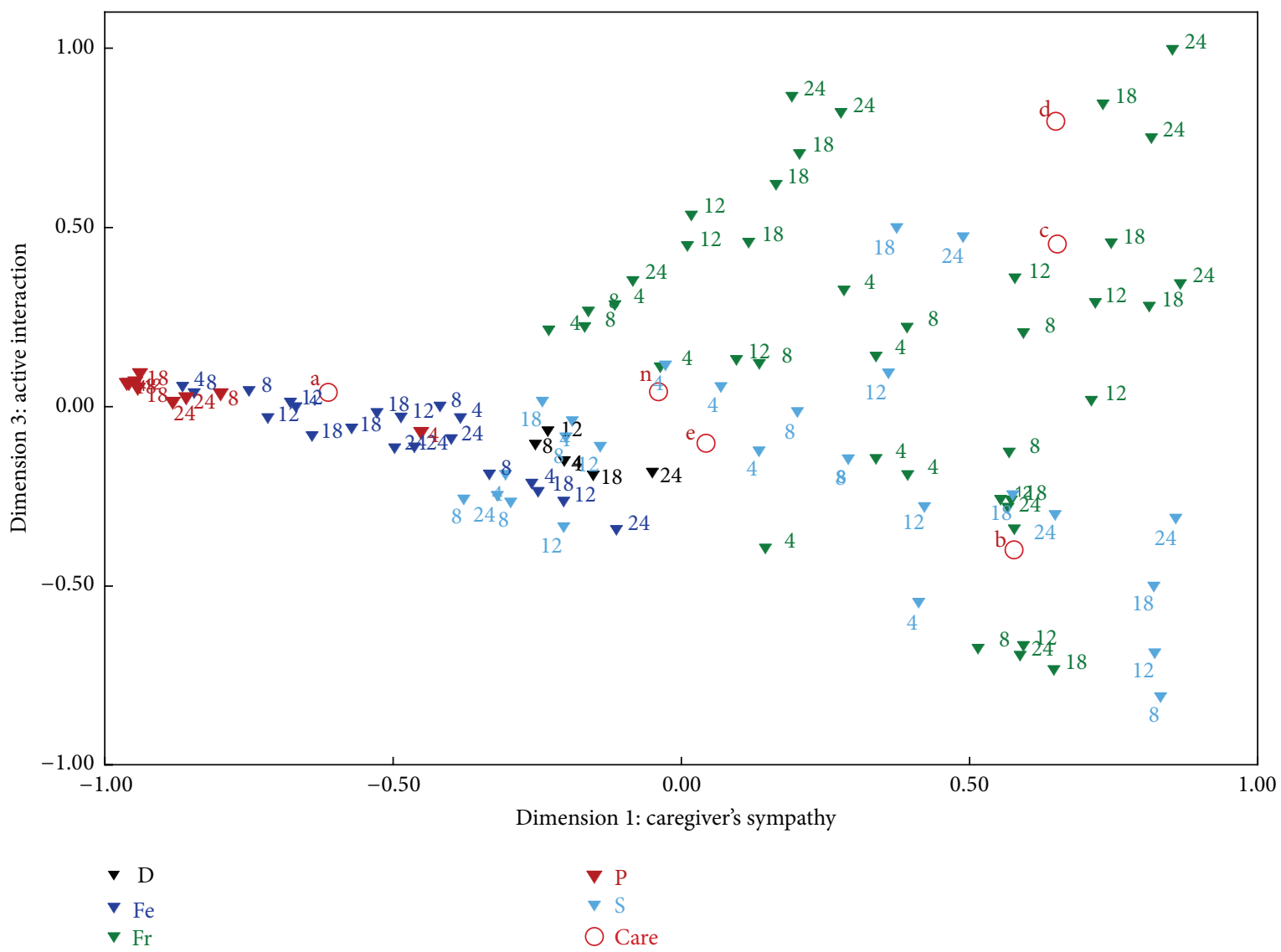

FiguRE 4: Correspondence analysis solution for soothing method and situations (Dimensions 1 \& 3). Note: D: discomfort; Fe: fear; Fr: frustration; P: physical pain; S: sadness; a: cuddling/giving love; b: distracting/attracting his/her attention to a novel object or event; c: giving drinks or snacks; d: waiting for the child to quiet down without assistance; e: not soothing the child because he/she does not cry or grumble; n: no answer. Each number indicates months of age.

soothing the child as he/she does not cry/grumble are at the higher pole. The rest of the soothing categories are at the lower pole. As the markers for 4 months are at the upper pole and those for 24 months are generally at the lower pole, the second dimension could reflect the children's biological maturity concerning engaging in situations. In Figure 4, the vertical direction (the third dimension) divides situations according to whether caregivers interact with infants actively. The lower pole reflects situations relating to active attempts to soothe, such as distracting, with some compassion, while the higher pole reflects situations relating to limited caregiverchild interaction, such as leaving the child as he or she is when he or she gets upset after asking for something and being told NO. Thus, distraction and ignoring or turning away are located at the lower and higher poles of the third dimension, respectively.

\section{Discussion}

In accord with recent discussions of the primary role of orienting as a control method in early life $[4,10]$, Figure 2 shows a strong correlation between parental reports of orienting in infancy and later reports of their child's Effortful Control.
In an effort to understand the effect of interchanges with caregivers on the early development of temperament, we longitudinally examined the soothing behaviors used by caregivers as well as rough-and-tumble play along with three dimensions of infant or toddler temperament: Positive Emotionality/Surgency, Negative Emotionality, and Orienting/Effortful Control. Consistent with prior studies, we found theoretical and conceptual continuity in temperamental factors across the IBQ-R and ECBQ data (Figure 2). We also found interchange effects of caregivers and infants on the development of Positive and Negative Emotionality at 24 months. Contrary to our expectations, we found no effects of distracting soothing methods or rough-and-tumble play on the development of the temperament-regulating capacity or Effortful Control, at least at 2 years of age.

As mentioned above, caregivers' frequent use of distracting behavior during infancy (Figure 2) is associated with higher Negative Affect in toddlers at 24 months. As Figure 4 suggests, the technique of distracting or attracting the child's attention to a novel object or event (choice b) is linked to situations of frustration, in which infants who have been more frequently soothed by distracting methods may have experienced frustration more often. Through experiences in which things are not going as the child expects, he or she may 
learn how to regulate his or her feelings. Age of 24 months is the so-called "terrible twos," during which toddlers often express powerful raw emotions directly. It is reported that the peak of temper tantrums is at 17-24 months [37]. One possibility is that strong negative feeling at 24 months may be related to later self-regulation or high Effortful Control, since Kopp [38] noted that the peak of negative behavior occurs at "about the time of heightened consciousness of self."

The above conclusion may be supported by the contribution of Surgency to both cuddling or giving love (choice a) and distracting or attracting the child's attention to a novel object or event (choice b). That is, as infants with strong surgent tendencies become able to crawl and move about, they create opportunities for experiencing distress or frustration, which may bring about parents' soothing behaviors such as choices (a) or (b). As cuddling or giving love behaviors are also positively related to Negative Affects at 24 months, it is suggested that early soothing behaviors by caregivers raise Negative Affects rather than influence emotional regulation at 24 months. These results remind us that early components of Negative Affects such as anger, frustration, or fear predict later guilt or shame [39,40]. As Negative Affects and Effortful Control are two dimensions of temperament that mediate the internalization of moral behavior [12], looking at relations between them in older children would be worthwhile.

We found that positive emotionality in infancy elicits rough-and-tumble play with caregivers, which is linked to higher Surgency scores at 24 months. This is partly consistent with our previous longitudinal study of toddlers (from 18 months to 36 months), which found that frequency of tickling is positively correlated with high-intensity pleasure and attention focusing scores on the ECBQ [31]. However, the present study does not support the possibility that children involved in more rough-and-tumble play will show earlier and better development of frontal executive functions [30]. As the participants in previous studies of rough-and-tumble play (e.g., $[41,42])$ were preschoolers, and as this is the first attempt to investigate infants' rough-and-tumble play, it is possible that the present participants were too young for us to examine a possible effect of such play.

The current findings concerning soothing methods and situations (Figures 3 and 4) are consistent with previous reports that shifting attention toward an object may not be appropriate when infants are at the highest levels of distress [43]. Physiological pain (e.g., from an injection) or stimuli related to being startled (e.g., a wailing siren) are strongly linked to cuddling/giving love (choice a). Conversely, reorienting or self-distracting behavior was not effective at high levels of negative reactivity [18]. Regarding the third dimension, some situations that include objects such as toys seem to be spontaneously linked to the technique of distracting or attracting the child's attention to a novel object or event (choice b). Leaving the child as he or she is (choice d) applied less frequently in young infants and seems to be linked to situations where the infant is angry, consistent with Huebner and Izard [44]. To assess distracting as a soothing method, situations that cause some negative feelings in infants related to the interruption of ongoing tasks or to something the infant does not like may be applicable.
The present study examined whether connections between the orienting and executive networks are influenced by how infants are soothed by their mothers. At the same time, we investigated the effect of rough-and-tumble play on the early development of temperament. Although the results of our soothing questionnaires are consistent with previous studies (e.g., $[18,43,44])$, the hypothesized role of parental soothing in the emergence of Effortful Control was not supported, at least at 2 years old. However, our sample size was rather small, and a larger sample size would provide greater statistical power. Furthermore, future investigations should include not only parent-reported questionnaire data but also laboratory observations or naturalistic home observations as each of these methods has advantages as well as disadvantages [45]. Elucidating how infants and caregivers jointly contribute to the development of temperamental control could inform early interventions for children at risk of poor emotional or behavioral regulation.

\section{Conflict of Interests}

The authors declare that there is no conflict of interests regarding the publication of this paper.

\section{Acknowledgments}

The present study was supported by Grant-in-Aid (nos. 22530712, 25285185) for Scientific Research from the Ministry of Education, Culture, Sports, Science, and Technology of Japan. Special thanks go to all the infants and families who generously took part in this project. The authors also thank Takatoshi Ogino, Toshiyuki Iguchi, and Tomoko Seo for help with data collection.

\section{References}

[1] H. A. Ruff and M. K. Rothbart, Attention in Early Development, Oxford University Press, New York, NY, USA, 1996.

[2] M. I. Posner and J. Fan, "Attention as an organ system," in Topics in Integrative Neuroscience: From Cells to Cognition, J. R. Pomerantz, Ed., pp. 31-61, Cambridge University Press, New York, NY, USA, 2008.

[3] M. I. Posner and M. E. Raichle, Imaging of Mind, Scientific American Books, New York, NY, USA, 1994.

[4] M. K. Rothbart, B. E. Sheese, M. R. Rueda, and M. I. Posner, "Developing mechanisms of self-regulation in early life," Emotion Review, vol. 3, no. 2, pp. 207-213, 2011.

[5] M. K. Rothbart and D. Derryberry, "Development of individual differences in temperament," in Advances in Developmental Psychology, M. E. Lamb and A. L. Brown, Eds., vol. 1, pp. 3786, Lawrence Erlbaum, Hillsdale, NJ, USA, 1981.

[6] M. I. Posner, M. K. Rothbart, B. E. Sheese, and P. Voelker, "Control networks and neuromodulators of early development," Developmental Psychology, vol. 48, no. 3, pp. 827-835, 2012.

[7] J. Colombo, "The development of visual attention in infancy," Annual Review of Psychology, vol. 52, pp. 337-367, 2001.

[8] B. M. Hood, "Shifts of visual attention in the human infant: a neuroscientific approach," in Advances in Infancy Research, C. Rovee-Collier and and L. P. Lipsitt, Eds., vol. 9, pp. 163-216, Ablex, Norwood, NJ, USA, 1995. 
[9] M. H. Johnson, M. I. Posner, and M. K. Rothbart, "Components of visual orienting in early infancy: contingency learning, anticipatory looking, and disengaging," Journal of Cognitive Neuroscience, vol. 3, no. 4, pp. 335-344, 1991.

[10] M. I. Posner, M. K. Rothbart, B. E. Sheese, and P. Voelker, "Developing attention: behavioral and brain mechanisms," Advances in Neuroscience, vol. 2014, Article ID 405094, 9 pages, 2014.

[11] B. E. Sheese, M. K. Rothbart, M. I. Posner, L. K. White, and S. H. Fraundorf, "Executive attention and self-regulation in infancy," Infant Behavior and Development, vol. 31, no. 3, pp. 501-510, 2008.

[12] M. I. Posner and M. K. Rothbart, Educating the Human Brain, American Psychological Association, Washington DC, USA, 2007.

[13] B. M. Hood and J. Atkinson, "Disengaging visual attention in the infant and adult," Infant Behavior and Development, vol. 16, no. 4, pp. 405-422, 1993.

[14] M. Matsuzawa and S. Shimojo, "Infants' fast saccades in the gap paradigm and development of visual attention," Infant Behavior and Development, vol. 20, no. 4, pp. 449-455, 1997.

[15] T. M. Field, "Infant gaze aversion and heart rate during face-toface interactions," Infant Behavior and Development, vol. 4, no. 3, pp. 307-315, 1981.

[16] B. A. McConnell and S. E. Bryson, "Visual attention and temperament: developmental data from the first 6 months of life," Infant Behavior and Development, vol. 28, no. 4, pp. 537-544, 2005.

[17] M. K. Rothbart, H. Ziaie, and C. O’Boyle, "Self-regulation and emotion in infancy," in Emotion and Its Regulation in Early Development, N. Eisenberg and R. Fabes, Eds., pp. 7-23, JosseyBass, San Francisco, Calif, USA, 1992.

[18] C. A. Stifter and J. M. Braungart, "The regulation of negative reactivity: function and development," Developmental Psychology, vol. 31, no. 3, pp. 448-455, 1995.

[19] M. A. Gartstein and M. K. Rothbart, "Studying infant temperament via the Revised Infant Behavior Questionnaire," Infant Behavior and Development, vol. 26, no. 1, pp. 64-86, 2003.

[20] S. P. Putnam, M. A. Gartstein, and M. K. Rothbart, "Measurement of fine-grained aspects of toddler temperament: The Early Childhood Behavior Questionnaire," Infant Behavior and Development, vol. 29, no. 3, pp. 386-401, 2006.

[21] S. P. Putnam, L. K. Ellis, and M. K. Rothbart, "The structure of temperament from infancy through adolescence," in Advances in Research on Temperament, A. Eliasz and A. Angleitner, Eds., pp. 165-182, Pabst Science Publishers, Lengerich, Germany, 2001.

[22] S. Casalin, P. Luyten, N. Vliegen, and P. Meurs, “The structure and stability of temperament from infancy to toddlerhood: a one-year prospective study," Infant Behavior and Development, vol. 35, no. 1, pp. 94-108, 2012.

[23] A. Nakagawa and M. Sukigara, "Individual differences in disengagement of fixation and temperament: longitudinal research on toddlers," Infant Behavior and Development, vol. 36, no. 4, pp. 728-735, 2013.

[24] S. P. Putnam, M. K. Rothbart, and M. A. Gartstein, "Homotypic and heterotypic continuity of fine-grained temperament during infancy, toddlerhood, and early childhood," Infant and Child Development, vol. 17, no. 4, pp. 387-405, 2008.

[25] C. Harman, M. K. Rothbart, and M. I. Posner, "Distress and attention interactions in early infancy," Motivation and Emotion, vol. 21, no. 1, pp. 27-43, 1997.
[26] M. H. Bornstein, "How infant and mother jointly contribute to developing cognitive competence in the child," Proceedings of the National Academy of Sciences of the United States of America, vol. 82, no. 21, pp. 7470-7473, 1985.

[27] J. Panksepp, Affective Neuroscience: The Foundations of Human and Animal Emotions, Oxford University Press, New York, NY, USA, 1998.

[28] M. G. Ruddy and M. H. Bornstein, "Cognitive correlates of infant attention and maternal stimulation over the first year of life," Child Development, vol. 53, no. 1, pp. 183-188, 1982.

[29] C. S. Tamis-LeMonda and M. H. Bornstein, "Habituation and maternal encouragement of attention in infancy as predictors of toddler language, play, and representational competence," Child Development, vol. 60, no. 3, pp. 738-751, 1989.

[30] J. Panksepp, "Can PLAY diminish ADHD and facilitate the construction of the social brain?" Journal of the Canadian Academy of Child and Adolescent Psychiatry, vol. 16, no. 2, pp. 57-66, 2007.

[31] A. Nakagawa and M. Sukigara, "The effects of soothing techniques and rough-and-tumble play on early temperament," The Journal of Child Health, vol. 69, no. 5, pp. 657-665, 2010.

[32] A. Nakagawa and M. Sukigara, "How are cultural differences in the interpretation of infant behavior reflected in the Japanese revised infant behavior questionnaire?" Japanese Journal of Educational Psychology, vol. 53, no. 4, pp. 491-503, 2005.

[33] M. Sukigara, A. Nakagawa, and R. Mizuno, "Studying toddler temperament via the Japanese early childhood behavior questionnaire," Paper presented at the 71st meeting of the Japanese Psychological Association, Tokyo, Japan, 2007.

[34] K. V. Mardia, "Measures of multivariate skewness and kurtosis with applications," Biometrika, vol. 57, no. 3, pp. 519-530, 1970.

[35] K. V. Mardia, "Applications of some measures of multivariate skewness and kurtosis in testing normality and robustness studies," Sankhyā, vol. 36, no. 2, pp. 115-128, 1974.

[36] D. Tofighi and D. P. MacKinnon, "RMediation: an R package for mediation analysis confidence intervals," Behavior Research Methods, vol. 43, no. 3, pp. 692-700, 2011.

[37] C. B. Kopp, "Emotional distress and control in young children," in Emotion and Its Regulation in Early Development, R. N. Eisenberg and R. A. Fabes, Eds., vol. 55, pp. 33-46, Jossey-Bass, San Francisco, Calif, USA, 1992.

[38] C. B. Kopp, "Emotion-focused coping in young children: self and self-regulatory processes," in Coping and the Development of Regulation: New Directions for Child and Adolescent Development, E. A. Skinner and M. J. Zimmer-Gembeck, Eds., pp. 33-46, Jossey-Bass, San Francisco, Calif, USA, 2009.

[39] G. Kochanska, J. N. Gross, M. Lin, and K. E. Nichols, "Guilt in young children: development, determinants, and relations with a broader system of standards," Child Development, vol. 73, no. 2, pp. 461-482, 2002.

[40] M. K. Rothbart, S. A. Ahadi, and K. L. Hershey, “Temperament and social behavior in childhood," Merrill-Palmer Quarterly, vol. 40, pp. 21-39, 1994.

[41] J. L. Flanders, V. Leo, D. Paquette, R. O. Pihl, and J. R. Séguin, "Rough-and-tumble play and the regulation of aggression: an observational study of father-child play dyads," Aggressive Behavior, vol. 35, no. 4, pp. 285-295, 2009.

[42] E. Scott and J. Panksepp, "Rough-and-tumble play in human children," Aggressive Behavior, vol. 29, no. 6, pp. 539-551, 2003.

[43] L. B. Jahromi, S. P. Putnam, and C. A. Stifter, "Maternal regulation of infant reactivity from 2 to 6 months," Developmental Psychology, vol. 40, no. 4, pp. 477-487, 2004. 
[44] R. R. Huebner and C. E. Izard, "Mothers' responses to infants' facial expressions of sadness, anger, and physical distress," Motivation and Emotion, vol. 12, no. 2, pp. 185-196, 1988.

[45] M. K. Rothbart and J. A. Bates, “Temperament," in Handbook of Child Psychology: Social, Emotional, and Personality Development, W. Damon, R. M. Lerner, and N. Eisenberg, Eds., vol. 3, pp. 99-166, Wiley, New York, NY, USA, 2006. 

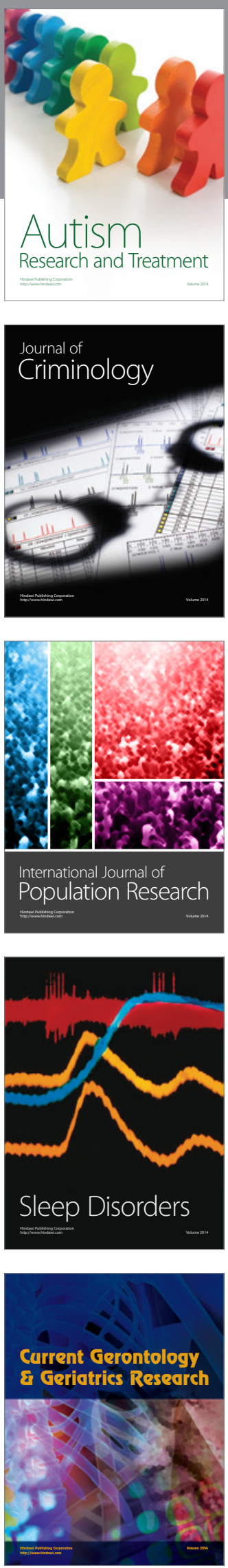
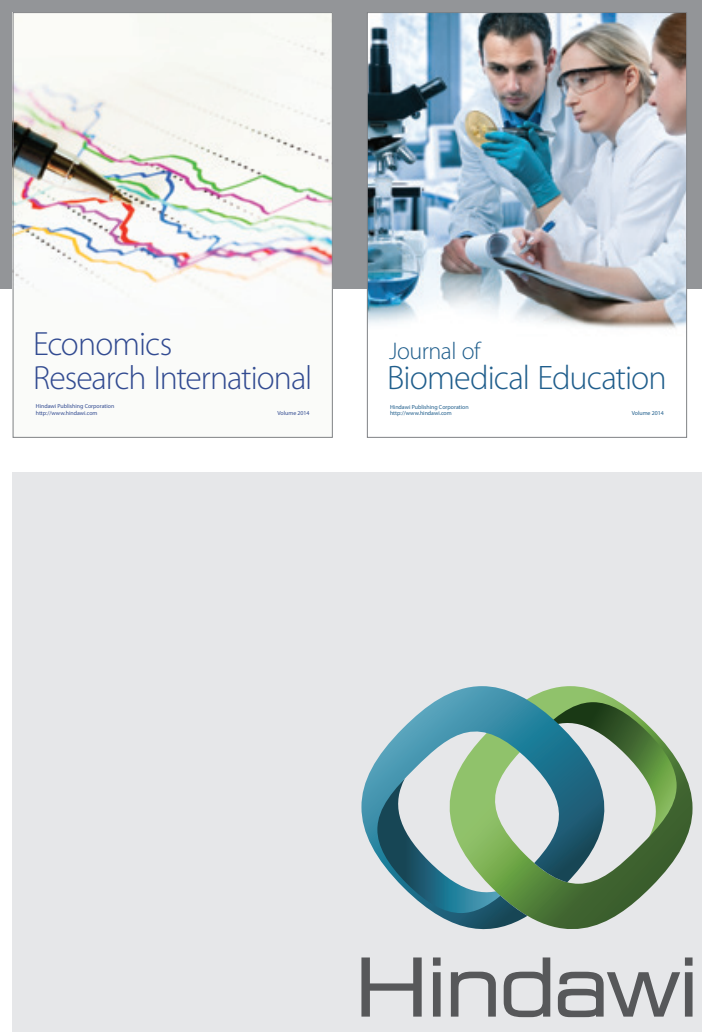

Submit your manuscripts at

http://www.hindawi.com
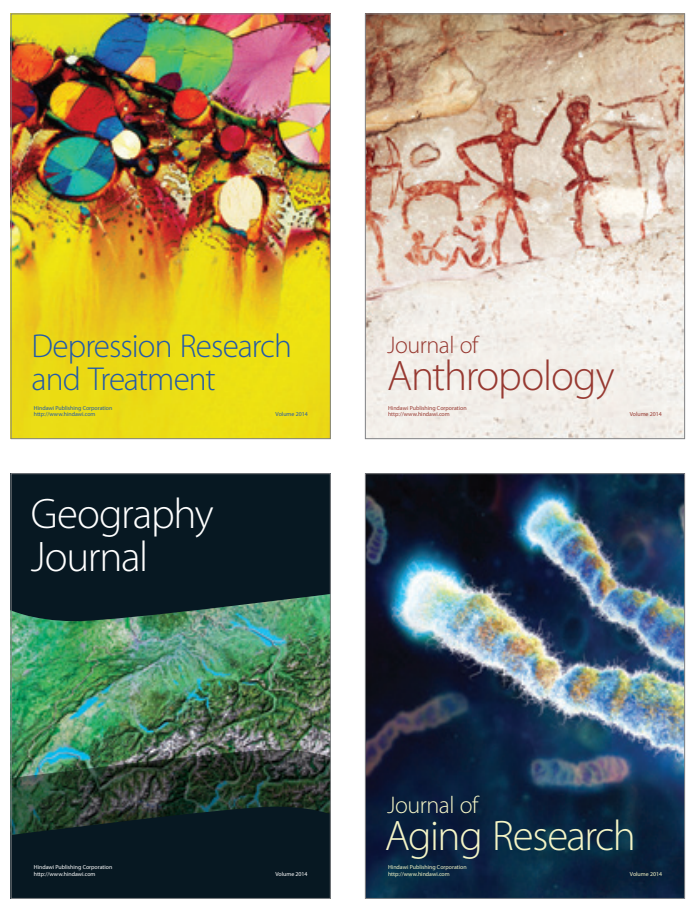
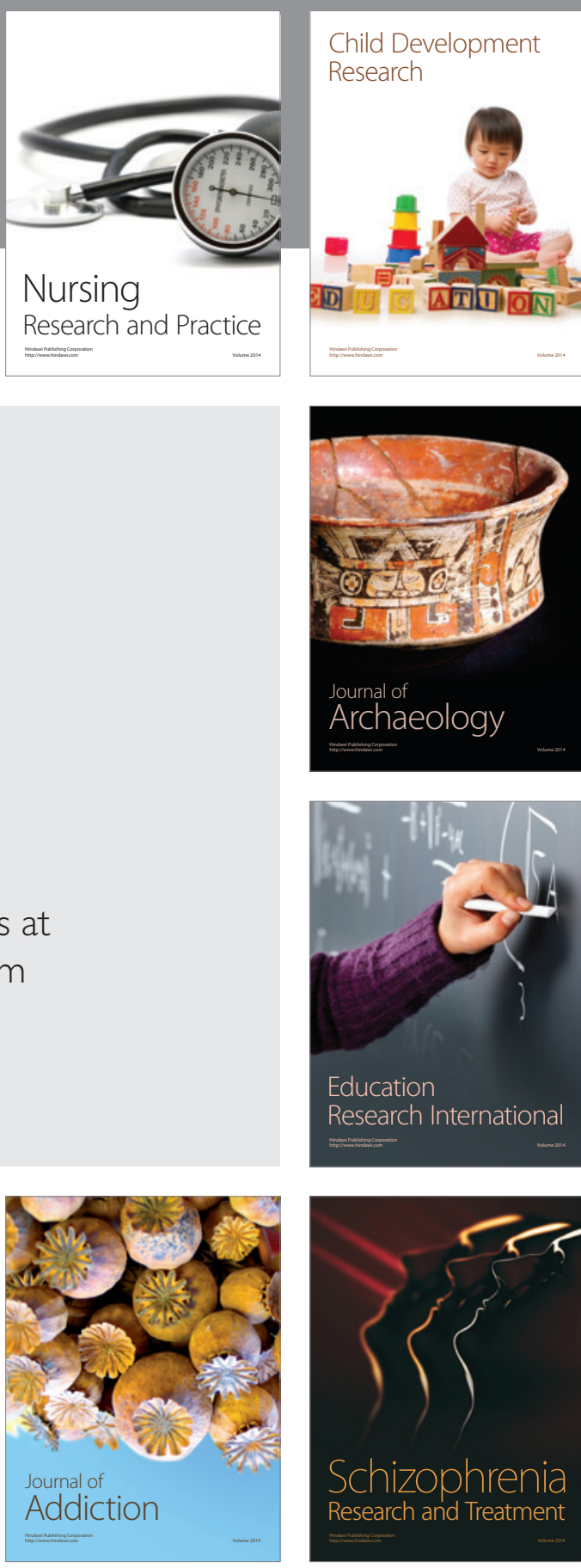

(D)
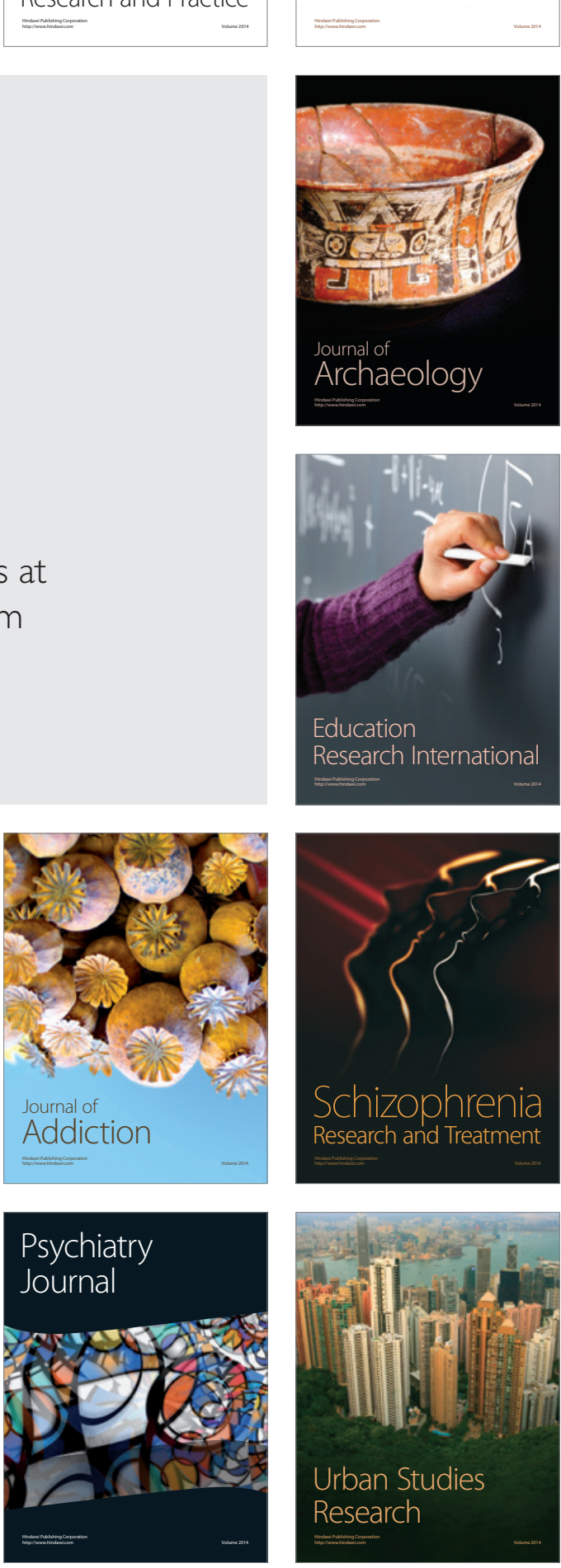